\title{
Assessment of some numerical methods for estimating the parameters of the one-dimensional advection-dispersion model
}

\author{
Davy D. Silavwe ${ }^{1} \cdot$ Isobel C. Brink ${ }^{1} \cdot$ Steve G. Wallis ${ }^{2}$
}

Received: 18 September 2018 / Accepted: 16 April 2019 / Published online: 30 April 2019

(c) The Author(s) 2019

\begin{abstract}
This study appraised optimisations of numerical solutions of the one-dimensional advection-dispersion model (AD-Model) to synthetic data generated using an analytical solution. The motivation for the work was to identify reliable methods for estimating stream solute transport parameters from observed events in small rivers. Numerical solutions of the AD-Model must contend with several effects that might disturb the solution, with the introduction of numerical diffusion and numerical dispersion being particularly important issues. This poses a problem if physical dispersion is being identified by optimising model coefficients using observations of solute transport from field experiments. The discretisation schemes used were the Backward-Time/Centred-Space, Crank-Nicolson, Implicit QUICK, MacCormack and QUICKEST methods. Optimisations were obtained for several grid resolutions by keeping the time step constant whilst varying the space step: the range of Peclet number, $P_{e}$, was 1.5-12.0. Generally, increasing the space step led to poorer estimated coefficients and poorer fits to the synthetic concentration profiles. For $P_{e}<5$ only Crank-Nicolson, MacCormack and QUICKEST gave reliable optimised dispersion coefficients: those from Backward-Time/Centred-Space and Implicit QUICK being significantly underestimated. For $P_{e}>5$ Crank-Nicolson and MacCormack gave slightly overestimated dispersion coefficients whilst the other methods gave significantly underestimated dispersion coefficients. These findings were generally consistent with the known presence of numerical diffusion and numerical dispersion in the methods.
\end{abstract}

Keywords Advection-dispersion model $\cdot$ Numerical methods $\cdot$ Solute transport parameters $\cdot$ Numerical properties . Optimisation

\section{Introduction}

Worldwide, there is a constant threat of high-level contamination of freshwater resources such as streams through, inter alia, discharge of effluents from treatment plants, accidental industrial spillage or intentional disposal of pollutants. In many cases, unintentional spills are the most significant economic danger to fresh water resources (USEPA 2009). Prediction of the subsequent movement and longitudinal spreading of contaminants in streams is necessary for a timely response by water authorities in respect of downstream

Steve G. Wallis

s.g.wallis@hw.ac.uk

1 Department of Civil Engineering, Stellenbosch University, Stellenbosch, Cape Town, South Africa

2 School of Energy, Geoscience, Infrastructure and Society, Heriot-Watt University, Riccarton, Edinburgh EH14 4AS, UK consumers and purposes of alleviation. Alleviation can only be possible if the characteristics of pollutant migration in such streams are reliably known. There are numerous processes that transport matter within streams. In the present context these processes can be categorised as either advection or mixing, and they exist in three spatial dimensions. However, we will assume they do not vary in time.

Attempting to include the processes in a 2- or 3-dimensional spatial framework would result in a complex model with difficulties associated with the availability of data for development of the model. A 1-dimensional model, conversely, has the advantages of improved ease of application as well as more easily obtainable data. However, all the mixing processes become combined in a single term, creating some separation between the model and the real world. Furthermore, the use of the 1-dimensional model is only suitable beyond an initial mixing zone, in which a pollutant becomes sufficiently well distributed within the cross section of the flow. Once achieved, the 1-dimensional mixing 
is usually termed longitudinal dispersion, represented by a dispersion coefficient (Fischer et al. 1979; Rutherford 1994; Martin and McCutcheon 1999; Ani et al. 2009), and the corresponding 1-dimensional advection is quantified by the cross-sectional average longitudinal velocity. Both of these vary with the flow rate and the size of the stream channel. Although stream geometry is usually variable along the stream, introducing significant complexities into the dispersive aspects (Ani et al. 2009), lack of data often prevents the inclusion of such detail and, therefore, streams are often modelled as uniform 1-dimensional systems. Consequently, application of the models produces reach-averaged solute transport parameter values (Wallis et al. 2013; Wallis and Manson 2004).

Most of the mass transport modelling in streams has been undertaken using the 1-dimensional governing transport equation known as the advection-dispersion model (ADModel) (Fischer et al. 1979; Rutherford 1994). It has been observed that best practices for estimating solute transport parameters combine tracer experiments with the AD-Model (Rutherford 1994). In this, workers combine solutions of the model equation with some form of parameter estimation. Experimental data is in the form of solute concentration-time profiles (or breakthrough curves), obtained by conducting slug-release tracer experiments. Several types of solution of the governing equation are available which include analytical solutions (Ogata and Banks 1961; Kumar et al. 2009) and numerical solutions (Abbott and Basco 1989; Versteeg and Malalasekera 2007).

In most practical situations the solution to governing equations is through numerical approaches because the initial and/or boundary conditions of analytical solutions are rarely satisfied in the field. There is evidence to suggest that, depending on the numerical method used, different solute transport parameter values can be estimated with the same observed data, such that prediction and interpretation of mass transport in streams may depend on the numerical approach used (Wallis and Manson 2004; Wallis et al. 2013). Numerical solution methods of the AD-Model must deal with several effects that disturb a solution. Primarily, these effects are a result of numerical approximation of the advective term (Sobey 1984; Abbott and Basco 1989), which may introduce artificial mixing (see later) into the solution. In the computed solution it may not be possible to differentiate between the artificial mixing introduced by a numerical scheme and the physical process of longitudinal dispersion (Sobey 1984). Therefore, it would be prudent to compare solutions given by various numerical methods. This is the theme of the current paper. The aim of the work was to investigate the reliability of several numerical methods for estimating stream solute transport parameters in a small stream using the AD-Model. The primary objective of the study was to estimate solute transport parameters using synthetic data generated with an analytical solution over a range of numerical grid resolutions. Since the parameter values used to generate the data were known, the accuracy of the estimated parameters could easily be assessed.

\section{Background}

The spreading of solutes in a fluid is termed as mixing (Chin 2013). Mixing occurs because of molecular diffusion, turbulent diffusion and shear dispersion. Longitudinal mixing in streams is primarily caused by shear dispersion which results from the stretching effect of cross-sectional velocity gradients combined with cross-sectional turbulent diffusion. The longitudinal dispersion coefficient is used to measure the longitudinal mixing of a solute which is well mixed across a channel (Chin 2013). Taylor (1954) argued that when a solute is well mixed in turbulent pipe flow longitudinal dispersion can be described by Fick's law. Based on this, Fischer argued that in open channels, sufficiently downstream of an initial mixing zone, concentration distributions can be modelled using an analogy of Fick's law (Fischer et al. 1979). In this zone there is an equilibrium between longitudinal velocity shear and transverse mixing. Depending on the release conditions of the solute, the zone may extend by up to 100 stream widths: Rutherford (1994) provides more detail on this issue. The general 1-dimensional equation describing longitudinal transport is expressed as (Rutherford 1994):

$\frac{\partial(A \varphi)}{\partial t}+\frac{\partial(A v \varphi)}{\partial x}=\frac{\partial}{\partial x}\left[D A \frac{\partial \varphi}{\partial x}\right]$

where $A$ is the cross-sectional area of the channel, $D$ is the longitudinal dispersion coefficient, $v$, is the cross-sectional average longitudinal velocity, $\varphi$, is the cross-sectional average solute concentration, $x$ is the longitudinal coordinate and $t$ is the time. The above equation is Taylor's (1954) and Fischer et al.'s (1979) 1-dimensional advection-dispersion model (AD-Model). Commonly, constant mixing rates and cross-sectional average velocities are assumed (Chanson 2004). Therefore, for constant dispersion coefficient and velocity, the 1-dimensional advection-dispersion model is:

$\frac{\partial(\varphi)}{\partial t}+v \frac{\partial(\varphi)}{\partial x}=D \frac{\partial^{2} \varphi}{\partial x^{2}}$

In this equation, the longitudinal dispersion coefficient quantifies the rate of longitudinal stretching of a solute cloud, and the cross-sectional average velocity quantifies the rate of downstream movement of the whole cloud (Chanson 2004; Wallis 2007). Application to a particular advection-dispersion problem requires a complete mathematical statement consisting of the AD-Model and specific boundary and initial conditions. The AD-Model is implemented through 
calibration, i.e. selection of values for coefficients $v$ and $D$ for use in solutions of Eq. (2), and several types of solution exist. The available solutions include analytical solutions (Ogata and Banks 1961; Kumar et al. 2009), numerical solutions (Wallis et al. 1998; Hoffman 2001; Manson et al. 2001; Chapra 2008) and routing procedures (Rutherford 1994; Singh and Beck 2003). There are very many solutions to the AD-Model depending on which boundary and initial conditions are specified (Fischer et al. 1979; Barnett 1983; Graf and Altinakar 1998; Chanson 2004; Chin 2013). Analytical approaches are derived to obtain solutions that are precise and continuous in time and space whereas numerical and routing approaches are inherently approximate and discrete.

There are essential analytical solutions of the AD-Model that are considered as the bases from which other solutions can be developed. These essential solutions mostly correspond to instantaneous slug releases of a solute in a stream in which the velocity and mixing fields are longitudinally uniform. One such solution is Taylor's solution of the ADModel with constant coefficients, written as (Rutherford 1994):

$\varphi(x, t)=\frac{M}{A \sqrt{4 \pi D t}} \exp \left[-\frac{(x-v t)^{2}}{4 D t}\right]$

where $M=$ mass of the solute released, and the other variables are as previously defined. The solution is based on the situation where concentration is known as a spatial distribution at an initial time, and it satisfies the following initial and boundary conditions (Rutherford 1994; Graf and Altinakar 1998):

$\varphi(x, 0)=M_{1} \delta(x)$

$\varphi(+\infty, t)=0$

$\varphi(-\infty, t)=0$

where $M_{1}$ is the mass of solute released per unit cross-sectional surface area and $\delta$ is the Dirac delta function. The Taylor solution is reliable in the equilibrium zone, i.e. at some distance downstream from the point where the slug is released (Rutherford 1994).

Equation (3) predicts Gaussian spatial concentration profiles and slightly skewed temporal concentration profiles. Observations of solute transport in streams are usually undertaken in the time domain and they too are not Gaussian, but they do not necessarily obey Taylor's solution (Chapra 2008). There are several reasons for this, e.g. the conditions in the field may not be consistent with the initial and boundary conditions of the solution, the stream may not be longitudinally uniform and the assumption of uniform concentration across the stream section may not hold. In addition, dead zones in the stream often create long tails in concentration profiles resulting in further deviations of temporal profiles from Gaussianity (Singh and Beck 2003).

Most practical situations require the use of a numerical solution because ultimately the models are required for more complex problems than those that allow the use of analytical solutions (Martin and McCutcheon 1999; Wallis 2007). Unfortunately, numerical solutions are neither exact nor continuous in both time and space, producing discrete solutions. Numerical solution techniques involve converting the governing differential equation into algebraic difference equations that can be solved for values at incremental points, or nodes, in space and time (Abbott and Basco 1989; Wallis 2007; Szymkiewicz 2010; Chapra and Canale 2015). In applying numerical methods, accurate results may be obtained when the number of nodes is infinitely large regardless of the method used. However, practical calculations can only use a limited number of nodes. In that case, results can only be physically realistic when the discretisation scheme has important properties, namely that they be stable, conservative, bounded and transportive (Ferziger and Peric 2002; Versteeg and Malalasekera 2007).

A solution method is said to stable if the errors that appear during the numerical solution process do not magnify; a conservative method is one that maintains the original mass of solute throughout the calculations. A bounded method entails that solutions remain within appropriate physical limits. Therefore, in the absence of sources, the concentration values at internal nodes should be bounded by boundary values, which in the context of solute transport in rivers implies that internal concentrations should be less than the concentrations propagated into the solution domain from the upstream boundary (because the physical system is advection dominated). A transportive method is one that recognises the directionality of influence in the flow. For solute transport in rivers conditions at a downstream location are heavily influenced by conditions further upstream with there being little or no influence from locations further downstream (because the physical system is advection dominated). In the numerical solution, the Peclet number (see below) plays an important role because it is a measure of the relative strength of advection and dispersion.

There are various discretisation schemes with varying properties (Leonard 1979; Abbott and Basco 1989; Hoffman 2001; Versteeg and Malalasekera 2007). Numerical solutions are discretised using one of the three basic approaches, namely: finite differences, finite volumes and finite elements (Abbott and Basco 1989; Ferziger and Peric 2002; Versteeg and Malalasekera 2007; Szymkiewicz 2010). However, the two most common discretisation approaches used in modelling solute transport in streams are the finite difference and finite volume methods. The finite volume approach has an advantage in that mass conservation is guaranteed (Versteeg and Malalasekera 2007) whereas finite difference schemes 
Fig. 1 Comparison of a simulated concentration profile using the Crank-Nicolson scheme with the corresponding physical concentration profile

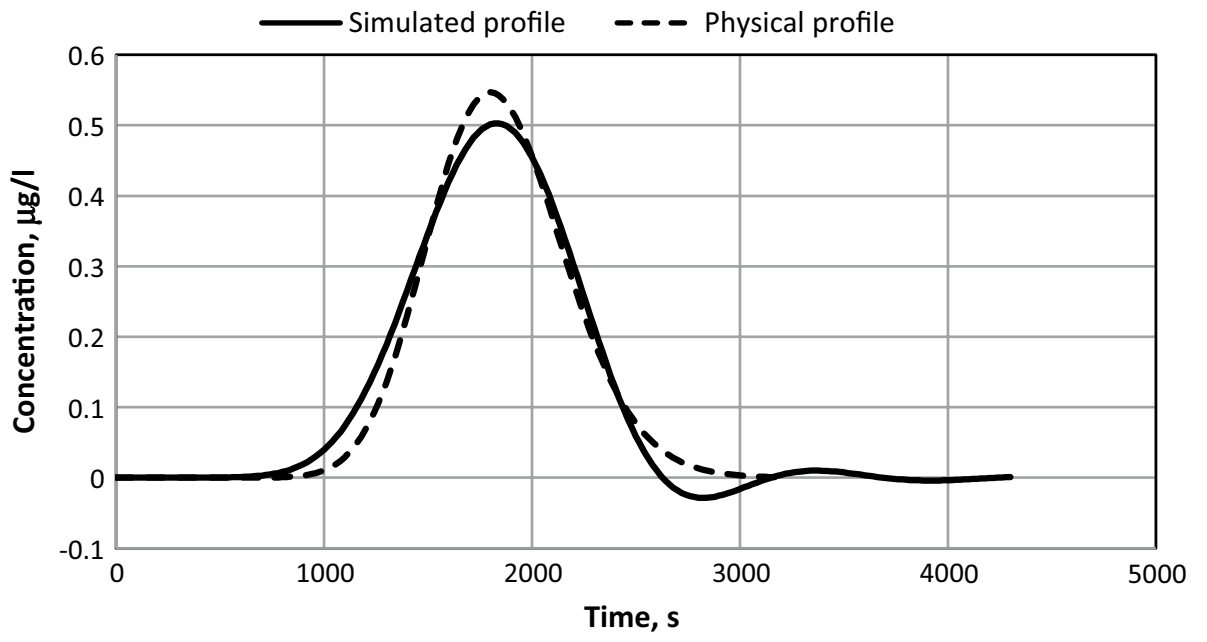

may require that special care is taken (Ferziger and Peric 2002). A solution method for a time-variable problem can be advanced in time in two ways, namely: explicitly and implicitly (see next section).

The main issue in the application of numerical methods to Eq. (2) is the formulation of an appropriate scheme for the values of the transported property when accounting for the advective contribution to the solution (Abbott and Basco 1989; Wallis and Manson 1997; Versteeg and Malalasekera 2007; Wallis 2007).

It is now necessary to define and explain some terminology in order to help mitigate some unavoidable confusion. Mathematically, Eq. (2) is of the form of an advection-diffusion equation. When the truncation errors of numerical solutions to it are examined, it is possible that they include the second, third and higher spatial derivatives of concentration. Mathematically, such terms introduce unwanted errors known as numerical diffusion (from the second spatial derivative) and numerical dispersion (from third spatial derivative). Similar errors are introduced by the higher spatial derivatives, but the second and third ones are the dominant terms. In the context of the application of Eq. (2) to longitudinal mixing in rivers the numerical diffusion enhances the physical dispersion causing greater longitudinal spreading and amplitude attenuation to occur in solutions than would be expected based on the value of the dispersion coefficient specified by the modeller (Chapra 2008; Szymkiewicz 2010). In contrast, numerical dispersion encourages the appearance of oscillations (or wiggles) in the numerical solutions (Leonard 1979; Manson and Wallis 1995; Chapra 2008) caused by the various components of the solution being propagated at different wave celerities (Szymkiewicz 2010). Usually the presence of these oscillations is most noticeable on the leading or trailing edge of a concentration profile and as a consequence the non-zero part of the profile extends further than it would if numerical dispersion were not present. In order to conserve the mass of the solute, some amplitude reduction and phase shift of the profile is often observed also. This is illustrated in Fig. 1 using a simulation with the Crank-Nicolson scheme (one of the schemes described later in the paper). Although it appears that the simulated concentration profile has spread further than the corresponding physical profile, the cause is not numerical diffusion, but is numerical dispersion. Since, physically, diffusion and dispersion are different processes, and dispersion has two quite different meanings to mathematicians and river modellers, in this paper we use the term artificial mixing when discussing the modified longitudinal spreading caused by any truncation error terms. Usually, the majority of this is due to numerical diffusion from the second spatial derivative in the truncation error.

The typical ways of judging the suitability of a numerical solution of the AD-Model, i.e. stability, boundedness, mass conservation etc., reveal little about the accuracy of a numerical solution (Sobey 1984). The fact that a scheme is numerically bounded or stable does not guarantee its accuracy. The grid resolution (i.e. the magnitude of the time and space steps in comparison with the duration and length, respectively, of the solute cloud being modelled) also has a considerable influence on the accuracy of a numerical scheme though it is often given little attention (Sobey 1984). Overall, the suitability of a numerical solution depends on the relative strength of the transport processes of advection and dispersion, the characteristics of the numerical method and the grid resolution used (Wallis and Manson 1997; Versteeg and Malalasekera 2007; Wallis 2007). Although these issues are inter-related the performance of a numerical method is heavily influenced by two non-dimensional properties known as the advection (or Courant) and dispersion (or diffusion) numbers. The ratio of the advection number to the dispersion number is known as the Peclet number (Ferziger and Peric 2002; Versteeg and Malalasekera 2007), 
which indicates whether the solution is dominated by advection or by dispersion (Chapra 2008; Chin 2013). An important application of the Peclet number is that it can be used to predict the appearance of oscillations (Abbott and Basco 1989; Versteeg and Malalasekera 2007; Szymkiewicz 2010).

In contrast to simulating a solute transport event, estimating solute transport parameters using observed concentration data requires an inverse modelling method. In this, values of model parameters are determined which give the best fit between the simulated and the observed data (Semuwemba 2011; Chin 2013). The level of agreement between the model output and the observations is used to assess the capability of the model (Runkel and Broshears 1991; Wallis et al. 2013), which can be assessed using one or more performance measures (Bennett et al. 2013).

\section{Applied numerical methods}

Several numerical methods were applied in this investigation. The choice of various discretisation schemes was based on discretisation type, solution method and scheme order. Hence, finite difference (FD) and finite volume (FV) discretisation approaches, explicit and implicit solution methods and first, second and third-order accurate discretisation schemes were chosen. The FD schemes were the BackwardTime/Centred-Space method, the Crank-Nicolson method and the MacCormack method (Ferziger and Peric 2002; Chapra 2008). The FV schemes were the Implicit QUICK (quadratic upstream interpolation for convective kinetics) method (Leonard 1979; Versteeg and Malalasekera 2007) and the QUICKEST (quadratic upstream interpolation for convective kinetics with estimated streaming terms) method (Leonard 1979).

The FD approach subdivides the solution domain into a mesh, in which grid lines serve as local coordinates. The derivatives in the AD-Model are expressed in terms of nodal quantities of both dependent and independent variables at the intersection of the grid lines. The discretisation results in algebraic equation(s) with all unknowns prescribed at discrete mesh points of the solution domain. The FV approach subdivides the solution domain into several control volumes $(\mathrm{CVs})$ each of which is centred at a node of the mesh. The derivatives in the AD-Model equation are integrated over the $\mathrm{CV}$ allowing the net solute mass entering the $\mathrm{CV}$ to be expressed in terms of the differences in advective and dispersive solute fluxes passing through the faces of the CV. The nodal values are then used in an interpolation formula to approximate the solute fluxes at the CV faces. Consequently, one obtains an algebraic equation for each $\mathrm{CV}$, in which several neighbouring nodal values appear (Ferziger and Peric 2002; Versteeg and Malalasekera 2007). "Appendix 1" provides explanatory figures for the two approaches.
A general approach for advancing a FD solution over time employs a temporal weighting, $\theta$, and is expressed as (Abbott and Basco 1989):

$$
\begin{gathered}
\frac{\varphi^{n+1}-\varphi^{n}}{\Delta t}+\left.(1-\theta) \frac{v \partial \varphi}{\partial x}\right|^{n}+\left.\theta \frac{v \partial \varphi}{\partial x}\right|^{n+1} \\
=\left.(1-\theta) \frac{D \partial^{2} \varphi}{\partial x^{2}}\right|^{n}+\left.\theta \frac{D \partial^{2} \varphi}{\partial x^{2}}\right|^{n+1}
\end{gathered}
$$

where $\Delta t$ is the time step and the superscripts $n$ and $n+1$ refer to the times at the start and end of the time step, respectively. Once the spatial gradients have been replaced by finite difference approximations, Eq. (7) is used to evaluate the solute concentration at time $n+1$ for all the nodes, assuming all nodal solute concentrations at time $n$ and all boundary conditions at the edges of the computational domain are known. When applying Eq. (7), if $\theta=0$ only transported variable values at the old time are used to evaluate one unknown concentration, resulting in an explicit calculation; if $\theta=1$, transported variable values at the new time level are used; and if $\theta=0.5$ transported variable values at both time levels are used. The latter two cases give a set of simultaneous equations containing all the unknown concentrations, resulting in an implicit calculation. In principle, the $\theta=0.5$ case is superior to the other cases because it is second-order accurate in time whilst the other cases are only first-order accurate in time. FV schemes can be expressed in a similar temporal weighting framework.

The non-dimensional numerical properties introduced earlier, which have an important bearing on the behaviour of the numerical schemes (advection number, dispersion number and Peclet number) are expressed as follows (Abbott and Basco 1989; Versteeg and Malalasekera 2007), where $\Delta x$ is the space step:

Advection number: $c=\frac{v \Delta t}{\Delta x}$

Dispersion number: $d=\frac{D \Delta t}{(\Delta x)^{2}}$

Peclet number: $P_{e}=\frac{v \Delta x}{D}$

Each of the numerical methods used is described in the following sub-sections. Information on the expected behaviour of the methods is considered later.

\section{The Backward-Time/Centred-Space method}

Using Eq. (7) with $\theta=1$, the method approximates the spatial derivatives at time level $n+1$ by the centred difference approach. Thus, the method is a Backward-Time/ 
Centred-Space Implicit scheme (Chapra 2008). The method is a FD scheme and is expressed as:

$\varphi_{j}^{n+1}=\varphi_{j}^{n}+\left[-v \frac{\varphi_{j+1}^{n+1}-\varphi_{j-1}^{n+1}}{2 \Delta x}+D \frac{\varphi_{j+1}^{n+1}-2 \varphi_{j}^{n+1}+\varphi_{j-1}^{n+1}}{(\Delta x)^{2}}\right] \Delta t$

For computational purposes, the above equation can be expressed in terms of the non-dimensional numerical properties introduced above as:

$-\left(d+\frac{c}{2}\right) \varphi_{j-1}^{n+1}+(1+2 d) \varphi_{j}^{n+1}-\left(d-\frac{c}{2}\right) \varphi_{j+1}^{n+1}=\varphi_{j}^{n}$

In this method values of the transported variable at the new time level are evaluated in terms of other unknown variable values at the new time level, requiring the solution of a set of simultaneous equations. This method is first-order accurate in time and second-order accurate in space and is unconditionally stable, allowing arbitrarily large time steps to be taken (Chapra 2008). In advection dominated flows Wallis (2007) warns that the promise of stability is outweighed by increasing inaccuracy as $\mathrm{c}$ increases above unity because the implicit nature of the method compromises the upstream-biased transportive nature of the physical system.

\section{The Crank-Nicolson method}

Using Eq. (7) with $\theta=0.5$ the method employs a centredtime/centred-space approach in which estimates of the spatial derivatives are expressed using values of the transported property at time levels $n$ and $n+1$. This is an implicit FD scheme and is expressed as:

$$
\begin{aligned}
\varphi_{j}^{n+1}= & \varphi_{j}^{n}+\frac{\Delta t}{2}\left[-v \frac{\varphi_{j+1}^{n+1}-\varphi_{j-1}^{n+1}}{2 \Delta x}+D \frac{\varphi_{j+1}^{n+1}+\varphi_{j-1}^{n+1}-2 \varphi_{j}^{n+1}}{\Delta x^{2}}\right] \\
& +\frac{\Delta t}{2}\left[-v \frac{\varphi_{j+1}^{n}-\varphi_{j-1}^{n}}{2 \Delta x}+D \frac{\varphi_{j+1}^{n}+\varphi_{j-1}^{n}-2 \varphi_{j}^{n}}{\Delta x^{2}}\right]
\end{aligned}
$$

For computational purposes the above equation can be written as:

$$
\begin{gathered}
-\left(\frac{d}{2}+\frac{c}{4}\right) \varphi_{j-1}^{n+1}+(d+1) \varphi_{j}^{n+1}-\left(\frac{d}{2}-\frac{c}{4}\right) \varphi_{j+1}^{n+1} \\
=\left(\frac{d}{2}+\frac{c}{4}\right) \varphi_{j-1}^{n}+(1-d) \varphi_{j}^{n}+\left(\frac{d}{2}-\frac{c}{4}\right) \varphi_{j+1}^{n}
\end{gathered}
$$

In this method, values of the transported variable at the new time level are evaluated in terms of variable values from both the old and the new time levels, requiring the solution of a set of simultaneous equations. The scheme is based on centred differencing in time and space and is thus second-order accurate in time and space. The scheme is unconditionally stable, but it has been observed to be inaccurate at high values of $P_{e}$ (Chapra 2008) and its implicit nature implies that the warnings of Wallis (2007) apply to it.

\section{The Implicit QUICK method}

This is a FV approach with CV face values of the transported variable expressed in terms of an upstream weighted parabolic interpolation and spatial gradients of the transported variable expressed using linear interpolation (Leonard 1979). Using $\theta=1$ in the FV equivalent version of Eq. (7) gives:

$$
\begin{aligned}
\varphi_{j}^{n+1}-\varphi_{j}^{n}= & -\frac{\Delta t v}{\Delta x}\left(\varphi_{j+1 / 2}^{n+1}-\varphi_{j-1 / 2}^{n+1}\right) \\
& +\frac{\Delta t D}{\Delta x}\left[\left(\frac{\partial \varphi}{\partial x}\right)_{j+1 / 2}^{n+1}-\left(\frac{\partial \varphi}{\partial x}\right)_{j-1 / 2}^{n+1}\right]
\end{aligned}
$$

Using Hayase et al.'s formulation (Hayase et al. 1992; Versteeg and Malalasekera 2007) to express variable values at the CV surfaces gives:

$\varphi_{j+1 / 2}^{n+1}=\varphi_{j}^{n+1}+\frac{1}{8}\left(3 \varphi_{j+1}^{n+1}-2 \varphi_{j}^{n+1}-\varphi_{j-1}^{n+1}\right)$

$\varphi_{j-1 / 2}^{n+1}=\varphi_{j-1}^{n+1}+\frac{1}{8}\left(3 \varphi_{j}^{n+1}-2 \varphi_{j-1}^{n+1}-\varphi_{j-2}^{n+1}\right)$

The discretised equation for a general FV centred at node, $j$, is expressed as:

$$
\begin{aligned}
& \frac{c}{8} \varphi_{j-2}^{n+1}-\left(d+\frac{7}{8} c\right) \varphi_{j-1}^{n+1}+\left(1+2 d+\frac{3}{8} c\right) \varphi_{j}^{n+1} \\
& \quad-\left(d-\frac{3}{8} c\right) \varphi_{j+1}^{n+1}=\varphi_{j}^{n}
\end{aligned}
$$

The scheme is first-order accurate in time and third-order accurate in space. Its implicit nature offers unconditional stability, but the warnings of Wallis (2007) apply to it.

\section{The MacCormack method}

The MacCormack (or Predictor-Corrector) method is a two-step FD method, unlike the above-discussed techniques which are one-step methods. There are various formulations of the approach (e.g. MacCormack 1982; Fürst and Furmánek 2011). Here we follow the semi-implicit formulation described in (Chapra 2008). The first step (predictor) uses the following explicit estimator, which uses forward spatial differencing for the advective term and centred spatial differencing for the dispersion term: 
$\varphi^{*}=\left(\frac{\varphi_{j}^{n+1}-\varphi_{j}^{n}}{\Delta t}\right)=-v \frac{\varphi_{j+1}^{n}-\varphi_{j}^{n}}{\Delta x}+D \frac{\varphi_{j+1}^{n}-2 \varphi_{j}^{n}+\varphi_{j-1}^{n}}{\Delta x^{2}}$

The second step (corrector) uses the following implicit estimator, which uses backward spatial differencing for the advective term and centred spatial differencing for the dispersion term:

$\varphi^{* *}=\left(\frac{\varphi_{j}^{n+1}-\varphi_{j}^{n}}{\Delta t}\right)=-v \frac{\varphi_{j}^{n+1}-\varphi_{i-1}^{n+1}}{\Delta x}+D \frac{\varphi_{j+1}^{n+1}-2 \varphi_{j}^{n+1}+\varphi_{j-1}^{n+1}}{\Delta x^{2}}$

Finally, an average of the two estimators is used to obtain the result expressed as:

$\varphi_{j}^{n+1}=\varphi_{j}^{n}+\left(\frac{\varphi^{*}+\varphi^{* *}}{2}\right) \Delta t$

This method, in a similar way to the Crank-Nicolson method, uses an average of an explicit scheme and an implicit scheme. For computational purposes, Eq. (21) can be expressed as:

$$
\begin{gathered}
-\left(\frac{d}{2}+\frac{c}{2}\right) \varphi_{j-1}^{n+1}+\left(1+d+\frac{c}{2}\right) \varphi_{j}^{n+1}-\frac{d}{2} \varphi_{j+1}^{n+1} \\
=\frac{d}{2} \varphi_{j-1}^{n}+\left(1+\frac{c}{2}-d\right) \varphi_{j}^{n}+\left(\frac{d}{2}-\frac{c}{2}\right) \varphi_{j+1}^{n}
\end{gathered}
$$

This has the same form as other implicit methods described above and is similar to that presented by Fürst and Furmánek (2011). The scheme is second-order accurate in time and space. Chapra (2008) claims that the scheme is conditionally stable, but Fürst and Furmánek (2011) claim it is unconditionally stable.

\section{The QUICKEST method}

The QUICKEST method (Leonard 1979) is a FV approach similar to, but superior to, the QUICK method. As well as using the upstream weighted parabolic interpolation of the QUICK method (Abbott and Basco 1989; Versteeg and Malalasekera 2007) it is an explicit formulation, using the $\theta=0$ version of the FV equivalent of Eq. (7). In addition, it includes 'estimated streaming terms' (EST) to account for advection and dispersion occurring during the time step (Leonard 1979). Variable values at CV faces are given by the following expressions (Leonard 1979; Abbott and Basco 1989):

$$
\begin{gathered}
\varphi_{j+1 / 2}^{n}=\frac{1}{2}\left(\varphi_{j}^{n}+\varphi_{j+1}^{n}\right)-\frac{1}{6}\left(\varphi_{j-1}^{n}-2 \varphi_{j}^{n}+\varphi_{j+1}^{n}\right) \\
\varphi_{j-1 / 2}^{n}=\frac{1}{2}\left(\varphi_{j-1}^{n}-\varphi_{j}^{n}\right)-\frac{1}{6}\left(\varphi_{j-2}^{n}-2 \varphi_{j-1}^{n}+\varphi_{j}^{n}\right)
\end{gathered}
$$

For computational purposes the method may be expressed as (Leonard 1979; Abbott and Basco 1989):

$$
\begin{aligned}
\varphi_{j}^{n+1}= & {\left[d(c)+\frac{c}{6}\left(c^{2}-1\right)\right] \varphi_{j-2}^{n} } \\
& +\left[d(1-3 c)-\frac{c}{2}\left(c^{2}-c-2\right)\right] \varphi_{j-1}^{n} \\
& -\left[d(2-3 c)-\frac{c}{2}\left(c^{2}-2 c-1\right)-1\right] \varphi_{j}^{n} \\
& +\left[d(1-c)-\frac{c}{6}\left(c^{2}-3 c+2\right)\right] \varphi_{j+1}^{n}
\end{aligned}
$$

This shows that unknown variable values at the new time level can be computed one at a time (an explicit calculation). In principle, the scheme is third-order accurate, but its explicit nature leads to conditionally stability. Thus, it can be unstable at modest values of $P_{e}$ : Leonard (1979) provides some detail on this issue.

\section{Expected behaviour of applied numerical methods}

Although the order of accuracy, given above, of the numerical methods gives an insight into how their performances might compare, a deeper study of the truncation error is much more revealing. This is achieved by substituting Taylor series expansions into the algorithms and identifying any temporal and spatial derivatives that might be a source of poor performance. As introduced earlier, the second and third spatial derivatives are particularly problematic so that methods which minimise the magnitude of such terms, or better still eliminate them, are likely to be among the better performing methods. Since the lowest spatial derivative emanating from the physical dispersion term is the 4th one it is only necessary to examine the truncation terms from the temporal and advective terms in order to quantify the numerical diffusion and numerical dispersion (from the dominant terms) of a numerical method. It is also necessary to replace any second or third temporal derivative with a corresponding spatial derivative, considering advection only. The procedure is often referred to as the modified equation approach (Szymkiewicz 2010) but appears elsewhere without being named (Abbott and Basco 1989).

Table 1 shows the results of this analysis for the five methods considered in the paper. "Appendix 2" summarises the method, provides intermediate expressions for all five methods and shows completed analyses for two of the schemes, namely Implicit QUICK (IQ) and MacCormack (M). Completed analyses for Crank-Nicolson $(\mathrm{CN})$ and Backward-Time/Centred-Space (BTCS) are readily available elsewhere (Szymkiewicz 2010). The reader is left to confirm the result for QUICKEST (Q) for themselves. In 
Table 1 Coefficients of numerical diffusion and numerical dispersion derived from the modified equation approach: $c$ is the advection number, as defined in Eq. (8)

\begin{tabular}{lll}
\hline Scheme & $\begin{array}{l}\text { Coefficient of numerical } \\
\text { diffusion }\end{array}$ & $\begin{array}{l}\text { Coefficient of numeri- } \\
\text { cal dispersion }\end{array}$ \\
\hline BTCS & $\Delta t \nu^{2} / 2$ & $-\Delta x^{2} \nu\left(-c^{2}+1\right) / 6$ \\
CN & 0 & $-\Delta x^{2} \nu\left(c^{2}+2\right) / 12$ \\
IQ & $\Delta t \nu^{2} / 2$ & $-\Delta x^{2} \nu\left(-c^{2}+1 / 4\right) / 6$ \\
M & 0 & $-\Delta x^{2} \nu\left(c^{2}+3 c+2\right) / 12$ \\
Q & 0 & 0 \\
\hline
\end{tabular}

the remainder of the paper we use the abbreviations introduced above to refer to the five numerical methods.

These results suggest that $\mathrm{CN}, \mathrm{M}$ and $\mathrm{Q}$ are free from numerical diffusion, but that BTCS and IQ contain the same amount of numerical diffusion, which depends only on the time step and the velocity. All the methods except $\mathrm{Q}$ contain numerical dispersion, but to differing degrees which are dependent on the space step, velocity and advection number. These results are consistent with those given elsewhere, e.g. Leonard (1979), Chapra (2008) and Szymkiewicz (2010).

\section{Computational procedure}

This study used Microsoft Excel spreadsheets to apply the numerical methods and followed the design described by Karahan $(2006,2007,2008)$. Excel spreadsheets have the advantages of computational speed and visual feedback through cell values and graphical displays (Billo 2007; Karahan 2006). Also, they can be configured to solve implicit numerical methods without the need for matrix algebra by writing algorithms in a direct form and using an iterative technique (Karahan 2006).

A separate workbook was created for each numerical method (Eqs. 12, 14, 18, 22 and 25). The general structure of the calculations for each implicit method is shown in Fig. 2. The input values were $\Delta x, \Delta t, v$ and $D$, and these were written to cells B1, B2, B3 and B4, respectively. The three numerical properties (Eqs. 8-10) were evaluated in cells B5, B6 and B7 and were derived from the input values. In the main calculation area columns represent different spatial locations or grid points (defined in row 8) and rows represent different times (defined in column $\mathrm{C}$ ). The time step was fixed, but the spatial resolution of the computational domain was varied so that model parameters could be estimated over a range of numerical properties. Each space step case had its own worksheet tab in each of the workbooks. Initial conditions were provided in row 10 (zero concentration at all locations), and the upstream boundary condition was provided in column E.

In each worksheet the direct form (see below) of the numerical method being tested was written to the cells in the main calculation area. Having entered the equation in cell F10, it was then copied along the row as far as a location well beyond the downstream end of the reach being modelled where a zero concentration downstream boundary condition was supplied. Provided that Excel's iterative

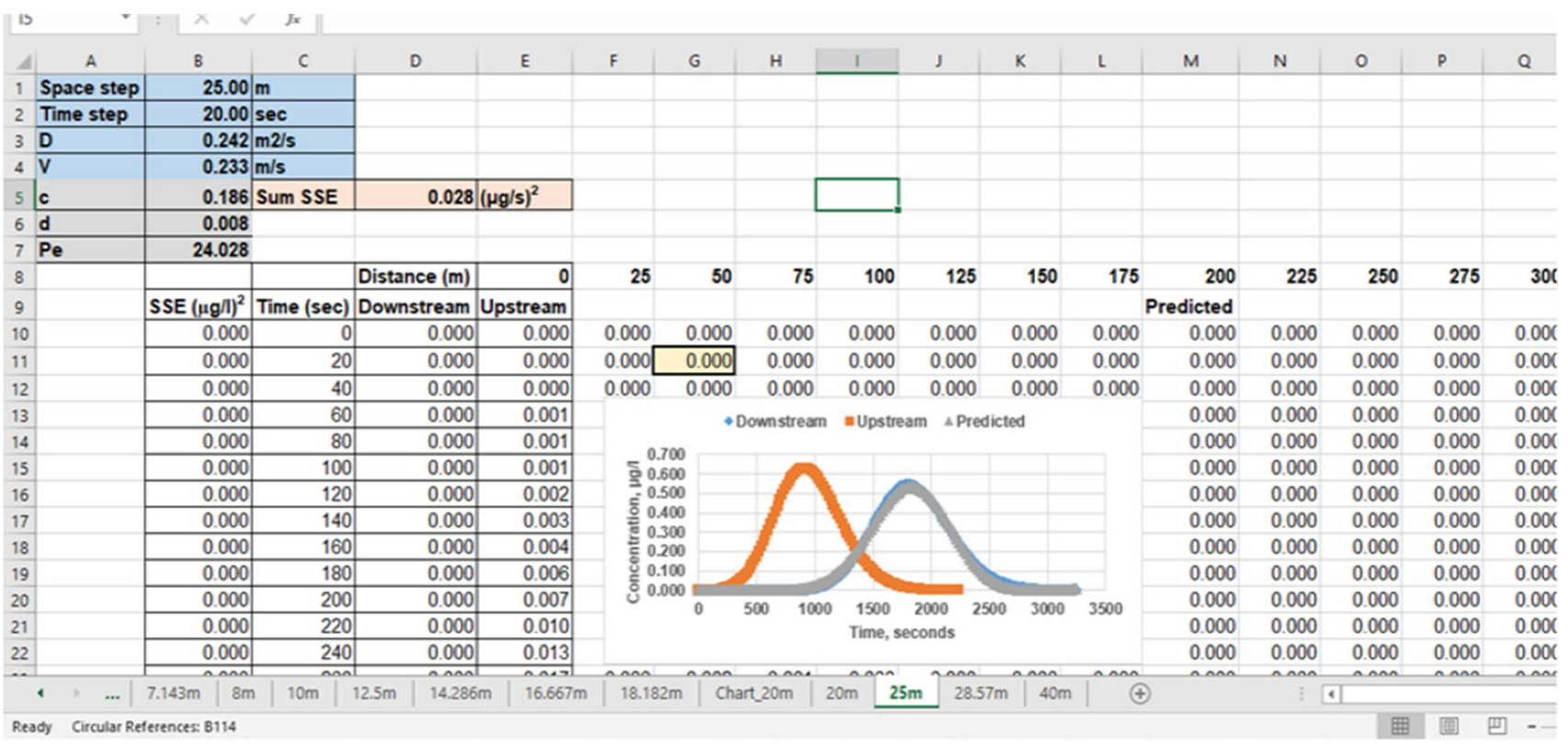

Fig. 2 Example spreadsheet showing the structure of the solution method (Backward-Time/Space-Centred method) for one space step case 
Fig. 3 Flow chart of the optimisation process

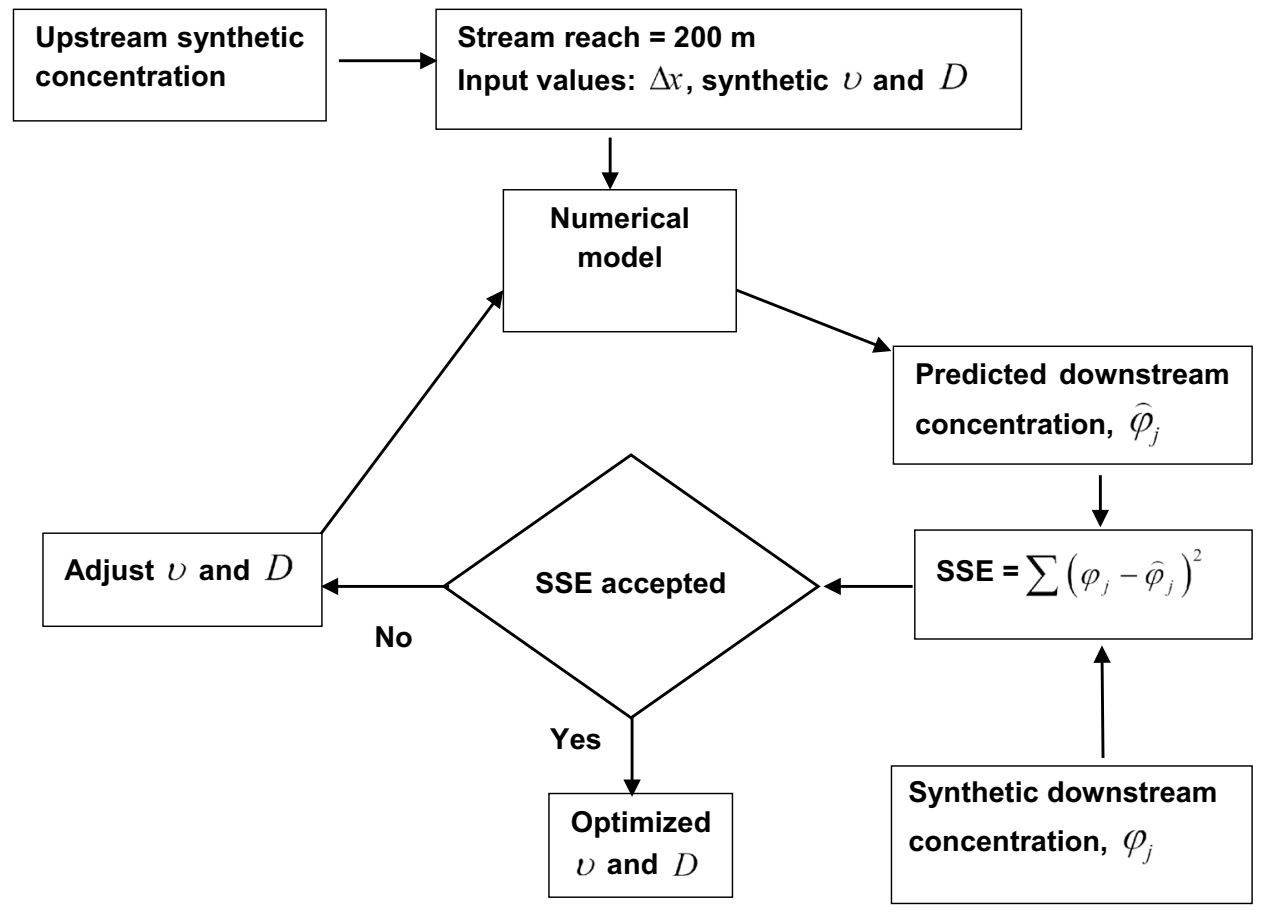

Implementation of the $\mathrm{Q}$ method followed the same spreadsheet design as used for the implicit methods except that an iterative solution of the direct form of the algorithm wasn't required (it being an explicit method an unknown concentration is only dependent on other concentration values in the previous row). In addition, however, a special treatment of the upstream boundary was needed because when applied to the cells in column F (see Fig. 2) a concentration value further upstream than the boundary is required. To overcome this, the $\mathrm{CN}$ scheme was applied to the cells in column F. Solving this simultaneously with the $\mathrm{Q}$ algorithm in column $\mathrm{G}$ yielded an explicit solution for column F. A similar $\mathrm{CN}$ implementation in column $\mathrm{F}$ was also used for the IQ method, but within the direct form of solution.

Some of the direct form implementations of the implicit methods did not always yield robust solutions (particularly higher Peclet number cases for BTCS). To remedy this, such cases were solved (also using Excel) using a double-sweep elimination method, which is described briefly in "Appendix 3". The opportunity was also taken to confirm a sample of the results from the other three implicit methods because the double-sweep elimination method could be applied to them with only a little extra work.

\section{Application of the numerical methods}

To assess the reliability of the numerical methods for identifying the velocity and the dispersion coefficient from temporal concentration profiles, synthetic data was (Lasdon et al. 1978; van den Bos 2007; Fylstra et al. 1998). Figure 3 summarises the procedure. 
Fig. 4 Variation of sum of squared errors (between simulations with optimised velocity and dispersion coefficient and synthetic downstream data) with Peclet number for the first data set

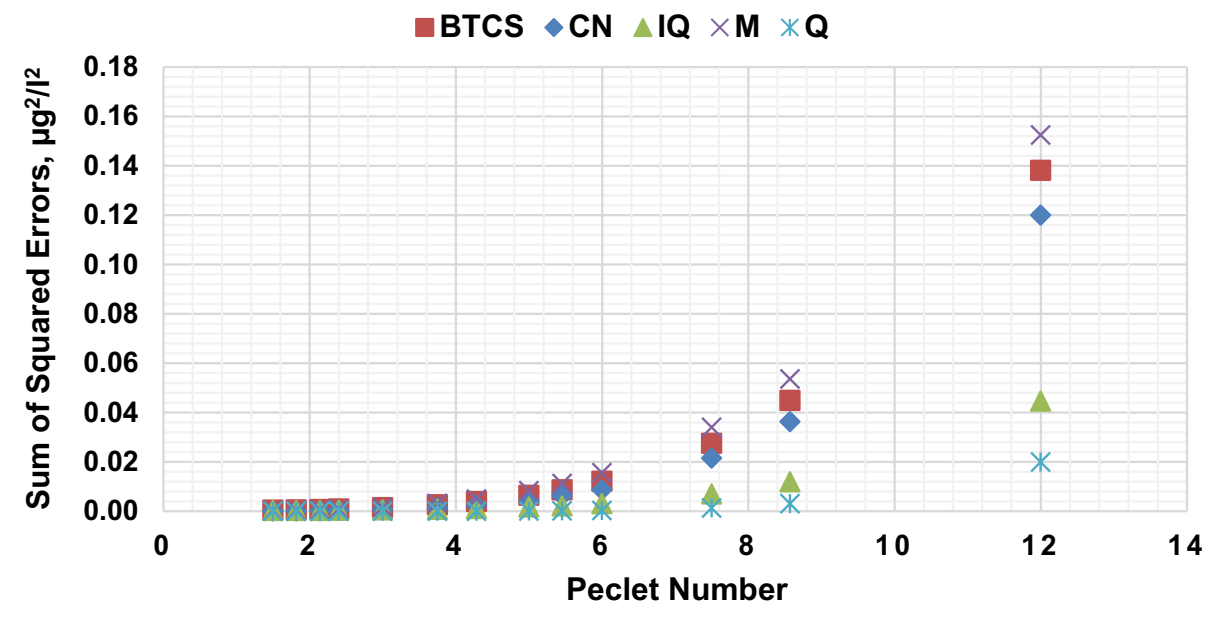

generated using Eq. (3). The use of synthetic data derived from analytical solutions has become common (Semuwemba 2011; Vaghela and Vaghela 2014) and has several advantages over using observed data. For example: conditions can be tailored for a particular situation, the values of solute transport parameters are known precisely and analytical solutions can provide results for a broad range of conditions that would not be practically possible with observed data (Semuwemba 2011). As well as attempting to estimate the parameter values used with the analytical solution, the numerical methods were also required to simulate the temporal concentration profiles given by the analytical solution at the downstream end of the reach.

Application of the numerical methods was made to synthetic data generated using two different sets of parameter values and time steps. The first set was generated using $v=0.225 \mathrm{~m} / \mathrm{s}$ and $D=0.750 \mathrm{~m}^{2} / \mathrm{s}$ with a time step of $20 \mathrm{~s}$ and the second set was generated using $v=0.150 \mathrm{~m} / \mathrm{s}$ and $D=0.500 \mathrm{~m}^{2} / \mathrm{s}$ with a time step of $30 \mathrm{~s}$. The parameter values were selected based on previous studies of the stream where the AD-Model was going to be applied in later research by the authors, namely the Murray Burn in Edinburgh, UK. A hypothetical stream reach of length $200 \mathrm{~m}$ was used, which was similar to the Murray Burn study reach length. Hence, for each synthetic data set an upstream and a downstream temporal concentration profile were generated at $600 \mathrm{~m}$ and $800 \mathrm{~m}$, respectively, from the solute source. The mass of solute was $1 \mathrm{~kg}$ and the crosssectional area of the channel was $1 \mathrm{~m}^{2}$. The numerical methods were applied to the synthetic data over a range of space steps $(5-40 \mathrm{~m})$, such that optimisation of the model parameters was observed under different values of the nondimensional numerical properties (e.g. $P_{e}$ being 1.5-12). The parameter values of the second set were deliberately chosen to yield the same values of the non-dimensional numerical properties as those for the first set.

\section{Results and discussion}

Results from the two sets of synthetic data were very similar in nature such that only one set of results is considered in detail here, namely that from the first set $(v=0.225 \mathrm{~m} / \mathrm{s}$, $D=0.750 \mathrm{~m}^{2} / \mathrm{s}$, time step $=20 \mathrm{~s}$ ). In all cases the numerical schemes behaved conservatively and simulations were stable. In several of the figures referred to below various aspects of the results are plotted against Peclet number, $P_{e}$. As shown previously, numerical discretisation can be expressed in terms of velocity, dispersion coefficient, space step and time step or in terms of advection number, dispersion number and Peclet number. The results were plotted against $P_{e}$ because it combines the effects of the other two non-dimensional properties and the plots are an effective way of showing the behaviour of the numerical methods when the space step is varied.

Figure 4 shows plots of the sum of squared errors between simulated and synthetic concentration profiles at the downstream end of the reach plotted against $P_{e}$ for the first data set. In every case the simulated profile was generated using the optimised velocity and dispersion coefficient. In general, simulation errors increased with increasing $P_{e}$ for all the numerical methods. Simulation errors obtained for $P_{e}<5$ were very small $\left(<0.01 \mu \mathrm{g}^{2} / \mathrm{l}^{2}\right)$ for all the methods, whilst for $P_{e}>5$ simulation errors increased significantly with increasing $P_{e}$ for all the methods. Generally, the finite difference methods gave higher simulation errors than the finite volume methods. The methods could be ranked in the following order of decreasing accuracy: Q, IQ, CN, BTCS, M. To a great extent this reflects the spatial orders of accuracy of the methods noted earlier. However, this is a rather superficial analysis to which some refinements are required, as described below.

Tables 2 and 3 show optimised values of velocity and dispersion coefficient. It should be noted that the values of the non-dimensional numerical properties, i.e. advection 
Table 2 Non-dimensional numerical properties and optimised velocities for first data set (synthetic velocity of $0.225 \mathrm{~m} / \mathrm{s})$

\begin{tabular}{lllllllll}
\hline$\Delta x(\mathrm{~m})$ & $\begin{array}{l}\text { Advection } \\
\text { number }\end{array}$ & $\begin{array}{l}\text { Dispersion } \\
\text { number }\end{array}$ & Peclet number & BTCS & CN & IQ & M & Q \\
\hline 5.00 & 0.900 & 0.600 & 1.500 & 0.226 & 0.225 & 0.226 & 0.226 & 0.225 \\
6.06 & 0.743 & 0.408 & 1.818 & 0.226 & 0.225 & 0.226 & 0.226 & 0.225 \\
7.14 & 0.630 & 0.294 & 2.143 & 0.226 & 0.226 & 0.226 & 0.226 & 0.225 \\
8.00 & 0.563 & 0.234 & 2.400 & 0.226 & 0.226 & 0.226 & 0.226 & 0.225 \\
10.00 & 0.450 & 0.150 & 3.000 & 0.227 & 0.226 & 0.226 & 0.227 & 0.225 \\
12.50 & 0.360 & 0.096 & 3.750 & 0.227 & 0.227 & 0.226 & 0.228 & 0.225 \\
14.29 & 0.315 & 0.073 & 4.286 & 0.228 & 0.227 & 0.227 & 0.228 & 0.225 \\
16.67 & 0.270 & 0.054 & 5.000 & 0.229 & 0.228 & 0.227 & 0.229 & 0.225 \\
18.18 & 0.248 & 0.045 & 5.454 & 0.229 & 0.229 & 0.227 & 0.230 & 0.225 \\
20.00 & 0.225 & 0.038 & 6.000 & 0.230 & 0.229 & 0.228 & 0.231 & 0.226 \\
25.00 & 0.180 & 0.024 & 7.500 & 0.233 & 0.232 & 0.229 & 0.234 & 0.226 \\
28.57 & 0.158 & 0.018 & 8.571 & 0.235 & 0.234 & 0.230 & 0.236 & 0.227 \\
40.00 & 0.113 & 0.009 & 12.000 & 0.243 & 0.242 & 0.235 & 0.244 & 0.231 \\
\hline
\end{tabular}

\begin{tabular}{lllllllll}
\hline$\Delta x(\mathrm{~m})$ & $\begin{array}{l}\text { Advection } \\
\text { number }\end{array}$ & $\begin{array}{l}\text { Dispersion } \\
\text { number }\end{array}$ & Peclet number & BTCS & CN & IQ & M & Q \\
\hline 5.00 & 0.900 & 0.600 & 1.500 & 0.235 & 0.749 & 0.255 & 0.749 & 0.746 \\
6.06 & 0.743 & 0.408 & 1.818 & 0.235 & 0.749 & 0.260 & 0.748 & 0.749 \\
7.14 & 0.630 & 0.294 & 2.143 & 0.234 & 0.748 & 0.264 & 0.747 & 0.750 \\
8.00 & 0.563 & 0.234 & 2.400 & 0.233 & 0.747 & 0.267 & 0.746 & 0.750 \\
10.00 & 0.450 & 0.150 & 3.000 & 0.231 & 0.746 & 0.273 & 0.745 & 0.749 \\
12.50 & 0.360 & 0.096 & 3.750 & 0.228 & 0.743 & 0.278 & 0.744 & 0.744 \\
14.29 & 0.315 & 0.073 & 4.286 & 0.226 & 0.742 & 0.279 & 0.744 & 0.739 \\
16.67 & 0.270 & 0.054 & 5.000 & 0.225 & 0.741 & 0.278 & 0.745 & 0.728 \\
18.18 & 0.248 & 0.045 & 5.454 & 0.225 & 0.750 & 0.275 & 0.747 & 0.718 \\
20.00 & 0.225 & 0.038 & 6.000 & 0.226 & 0.739 & 0.269 & 0.751 & 0.704 \\
25.00 & 0.180 & 0.024 & 7.500 & 0.238 & 0.752 & 0.240 & 0.770 & 0.649 \\
28.57 & 0.158 & 0.018 & 8.571 & 0.255 & 0.766 & 0.207 & 0.790 & 0.594 \\
40.00 & 0.113 & 0.009 & 12.000 & 0.316 & 0.827 & 0.032 & 0.854 & 0.332 \\
\hline
\end{tabular}

Table 3 Non-dimensional numerical properties and optimised dispersion coefficients for first data set (synthetic dispersion coefficient of $0.750 \mathrm{~m}^{2} / \mathrm{s}$ ) number, dispersion number and Peclet number, shown in the tables were calculated using the parameter values used for generating the synthetic data so that the results of all methods could be easily compared. It can be observed that there is variation of optimised velocity and dispersion coefficient both with the numerical method used and with the numerical properties. In all cases the optimised velocity is greater than the synthetic value. For $P_{e}<5$ the percentage errors in the velocities are typically less than $2 \%$. They increase to about $8 \%$ when $P_{e}=12$. Generally, Q has the smallest velocity errors and $\mathrm{M}$ has the largest.

Turning attention to the optimised dispersion coefficients, which generally show greater errors than the optimised velocities, Fig. 5 shows results for all the numerical methods for the first data set (i.e. the data in Table 3). It can be observed that for $P_{e}<5$, the results fall into two distinct groups. Here, CN, M and Q yield optimised dispersion coefficients that agree closely with the synthetic value; BTCS and IQ yield values that are similar to each other, but which are considerably too small. This is consistent with the analysis of the numerical diffusion in the methods shown in Table 1. Furthermore, the error in the optimised dispersion coefficients (i.e. the difference between the optimised value and that used to generate the synthetic data) for BTCS and IQ is very close to the value given by the numerical diffusion coefficient in the table $\left(\sim 0.5 \mathrm{~m}^{2} / \mathrm{s}\right.$, using the synthetic value of velocity). In other words, the presence of numerical diffusion means that a smaller physical dispersion coefficient than the synthetic value is required to fit the model to the data.

For $P_{e}>5$, optimised dispersion coefficients for all the methods diverge from the synthetic value. Those from $\mathrm{CN}, \mathrm{M}$ and BTCS show a small increase from the value in the lower $P_{e}$ range whilst those from IQ and Q show a 
Fig. 5 Variation of optimised dispersion coefficient with Peclet number for first data set (synthetic dispersion coefficient of $0.750 \mathrm{~m}^{2} / \mathrm{s}$-indicated by dashed line)
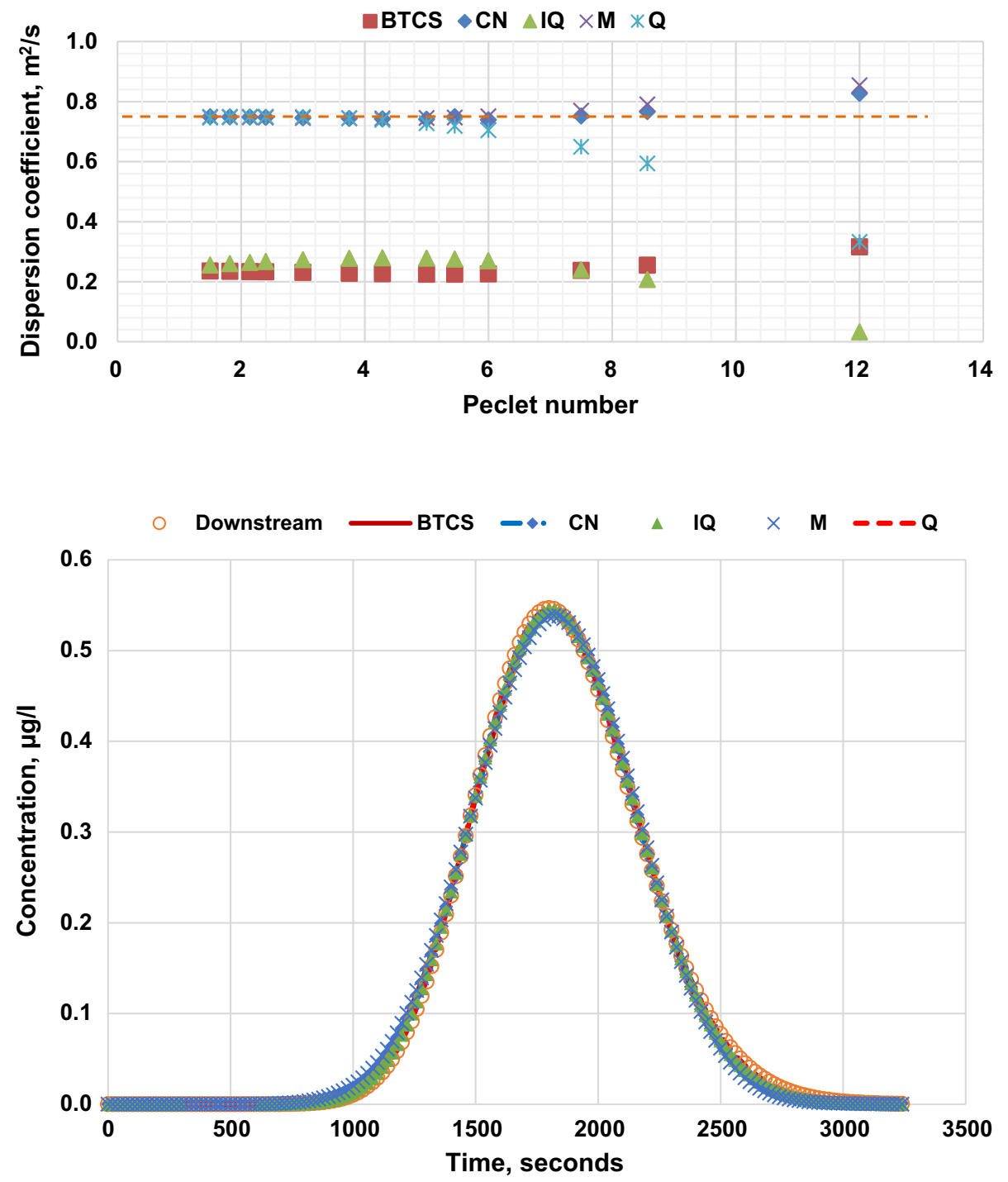

Fig. 6 Simulation results for five numerical methods using a space step of $20 \mathrm{~m}$ and a time step of $20 \mathrm{~s}$ for first data set (synthetic dispersion coefficient of $0.750 \mathrm{~m}^{2} / \mathrm{s}$, synthetic velocity of $0.225 \mathrm{~m} / \mathrm{s}$, Peclet number $=6.0$ ) substantial decrease from the value in the lower $P_{e}$ range. In both cases, the divergence increases with increasing $P_{e}$.

In order to aid the interpretation of these results, Figs. 6 and 7 show composite plots (i.e. results from all 5 methods) of the simulated concentration profiles for two values of $P_{e}$, namely, 6 and 12, respectively. The downstream synthetic concentration profile is also shown. For the lower $P_{e}$ case, there is little difference between the simulated profiles and they all match the synthetic profile well. This reflects the relatively low modelling errors shown in Fig. 4 (described earlier). In contrast, Fig. 7 shows that, to a greater or lesser extent, all 5 simulated profiles are distorted, being characterised by a reduced amplitude (compared to the synthetic profile), a little more spreading (particularly on the rising limb) and an oscillation on the trailing edge. Such behaviour is caused by numerical dispersion, as described earlier (see Fig. 1).
Table 4 shows the coefficients of numerical dispersion, obtained using the formulae in Table 1, for four values of $P_{e}$ from the range covered in the optimisations. Clearly, these coefficients increase with increasing $P_{e}$ for all schemes, and they are significantly larger for $P_{e}=12$ than for $P_{e}=6$. Furthermore, the degree of distortion of the simulated profiles in Fig. 7 (for the higher $P_{e}$ case) mirrors the magnitude of the numerical dispersion coefficients. Thus, the ranking order of the degree of distortion is similar to the ranking order of the coefficients of numerical dispersion. For example, the errors in the peak concentrations are $-8.6 \%,-9.2 \%$, $-12.0 \%,-12.4 \%,-13.5 \%$ for Q, IQ, CN, BTCS and M, respectively.

For $\mathrm{CN}$ and $\mathrm{M}$ in the higher $P_{e}$ range the generally larger dispersion coefficient than the synthetic value (see Fig. 5 and Table 3) helps to damp out the oscillation, but sacrifices the fit around the peak of the profile. Of course, the optimisation finds the combination of velocity and dispersion coefficient 
Fig. 7 Simulation results for five numerical methods using a space step of $40 \mathrm{~m}$ and a time step of $20 \mathrm{~s}$ for first data set (synthetic dispersion coefficient of $0.750 \mathrm{~m}^{2} / \mathrm{s}$, synthetic velocity of $0.225 \mathrm{~m} / \mathrm{s}$, Peclet number $=12.0$ )
Table 4 Coefficients of numerical dispersion evaluated using the formulae in Table 1

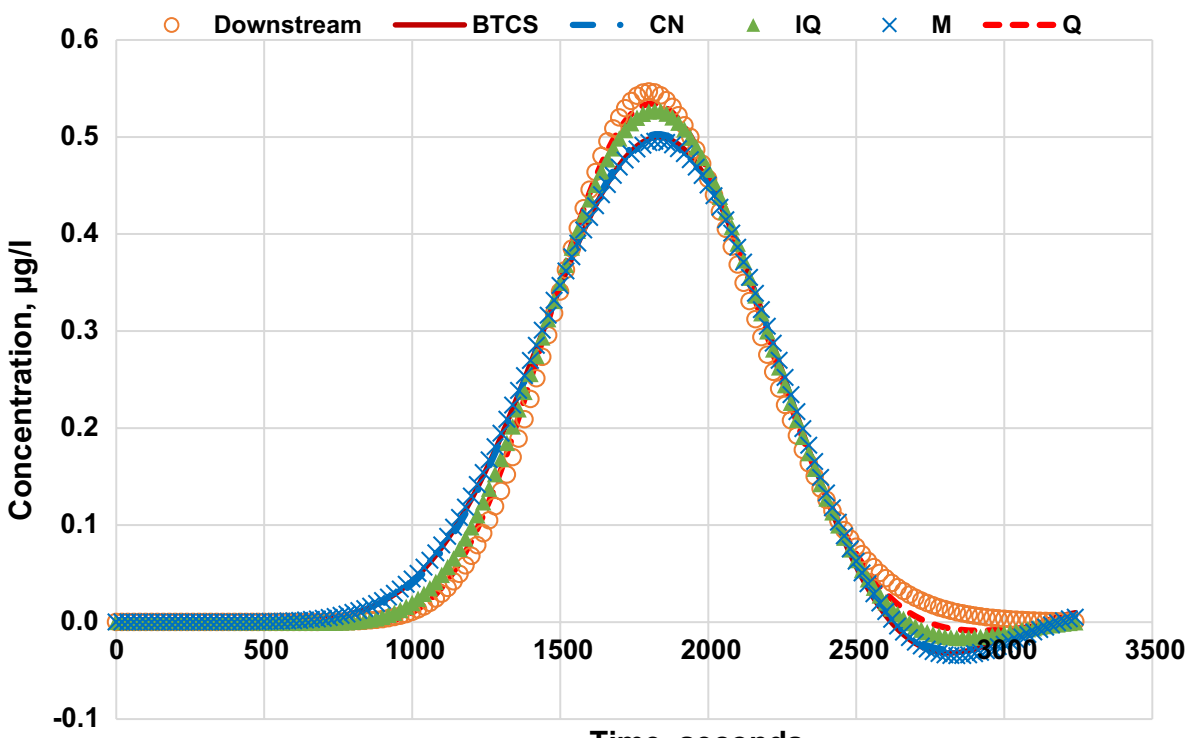

Time, seconds

\begin{tabular}{llllrrrrr}
\hline$\Delta x(\mathrm{~m})$ & $\begin{array}{l}\text { Advection } \\
\text { number }\end{array}$ & $\begin{array}{l}\text { Dispersion } \\
\text { number }\end{array}$ & Peclet number & BTCS & CN & IQ & M & Q \\
\hline 5.00 & 0.900 & 0.600 & 1.500 & -0.2 & -1.3 & 0.5 & -2.6 & 0 \\
10.00 & 0.450 & 0.150 & 3.000 & -3.0 & -4.2 & -0.27 & -6.8 & 0 \\
20.00 & 0.225 & 0.038 & 6.000 & -14.5 & -15.7 & -3.0 & -21.1 & 0 \\
40.00 & 0.113 & 0.009 & 12.000 & -63.8 & -65.0 & -14.8 & -77.6 & 0 \\
\hline
\end{tabular}

that gives the best fit considering the whole profile. In these cases where the optimised dispersion coefficient is larger than the synthetic value, artificial mixing arises from the fitting of the model to the distorted profile and so is related to the presence of numerical dispersion rather than numerical diffusion. Similarly for BTCS, and allowing for the numerical diffusion it contains, some additional physical dispersion (compared to the lower $P_{e}$ range) is helpful in damping out the oscillations in the higher $P_{e}$ range leading to a better fit to the whole concentration profile. Although the presence of numerical dispersion influences the optimised dispersion coefficient via model fitting to the distorted profile (similarly to $\mathrm{CN}$ and $\mathrm{M}$ ), in these BTCS cases artificial mixing is dominated by the presence of numerical diffusion.

The results from IQ and Q in the higher $P_{e}$ range display smaller oscillations than the other three methods which enables the optimisation to find better fits to the synthetic profile (see Fig. 4), but the dispersion coefficients are too small suggesting that additional numerical errors become significant in the higher $P_{e}$ range. Regarding Q, it is interesting that the profile in Fig. 7 shows a distortion, yet according to Table 1 the method contains no numerical dispersion. Similarly, the optimised dispersion coefficients are very small (implying that numerical diffusion is present), in contrast to the result in Table 1 . It seems very likely, therefore, that higher-order truncation error terms than the second and third spatial derivatives are playing a significant role. Such higher-order terms were not included in the modified equation analysis because usually the second and third spatial derivatives are the dominant terms for numerical diffusion and numerical dispersion, respectively. Since even truncation error terms are associated with numerical diffusion (Szymkiewicz 2010), the source of this numerical diffusion in $\mathrm{Q}$ is likely to be the 4th spatial gradient term. The trend of the IQ results in Fig. 5 in the higher $P_{e}$ range is similar to that of Q, reflecting their common basis (quadratic upstream interpolation of control volume face concentrations). So it seems that higher-order truncation error terms affect the IQ results also, but of course in this case numerical errors from the second and third spatial derivatives are also present (see Tables 1 and 4). However, the latter is much smaller than for the other implicit methods (see Table 4), as reflected in the modelling errors shown in Fig. 4.

IQ and Q may also be affected by the $\mathrm{CN}$ treatment of the first computational cell next to the upstream boundary. This will introduce a little numerical dispersion, and the effect will be greater for the higher $P_{e}$ case than for the lower $P_{e}$ case because (a) the $\mathrm{CN}$ method contains more numerical dispersion in the former than in the latter (see Table 4) and (b) there is a greater ratio of affected computational cells 
Table 5 Non-dimensional numerical properties and ratio of optimised dispersion coefficients to synthetic dispersion coefficient for the first data set

\begin{tabular}{lllllllll}
\hline$\Delta x(\mathrm{~m})$ & $\begin{array}{l}\text { Advection } \\
\text { number }\end{array}$ & $\begin{array}{l}\text { Dispersion } \\
\text { number }\end{array}$ & Peclet number & BTCS & CN & IQ & M & Q \\
\hline 5.00 & 0.900 & 0.600 & 1.500 & 0.314 & 0.999 & 0.340 & 0.998 & 0.995 \\
10.00 & 0.450 & 0.150 & 3.000 & 0.308 & 0.994 & 0.364 & 0.993 & 0.998 \\
20.00 & 0.225 & 0.038 & 6.000 & 0.301 & 0.985 & 0.359 & 1.001 & 0.939 \\
40.00 & 0.113 & 0.009 & 12.000 & 0.421 & 1.103 & 0.043 & 1.139 & 0.443 \\
\hline
\end{tabular}

\begin{tabular}{lllllllll}
\hline$\Delta x(\mathrm{~m})$ & $\begin{array}{l}\text { Advection } \\
\text { number }\end{array}$ & $\begin{array}{l}\text { Dispersion } \\
\text { number }\end{array}$ & Peclet number & BTCS & CN & IQ & M & Q \\
\hline 5.00 & 0.900 & 0.600 & 1.500 & 0.314 & 0.999 & 0.340 & 0.998 & 0.995 \\
10.00 & 0.450 & 0.150 & 3.000 & 0.308 & 0.994 & 0.364 & 0.993 & 0.998 \\
20.00 & 0.225 & 0.038 & 6.000 & 0.301 & 0.985 & 0.359 & 1.001 & 0.939 \\
40.00 & 0.113 & 0.009 & 12.000 & 0.421 & 1.103 & 0.043 & 1.139 & 0.443 \\
\hline
\end{tabular}

Table 6 Non-dimensional numerical properties and ratio of optimised dispersion coefficients to synthetic dispersion coefficient for the second data set models of the AD-Model unless due attention is paid to the characteristics of the numerical method and the numerical properties under which the models are applied. In particular, the presence of artificial mixing needs to be taken into account. Optimised dispersion coefficients will be underestimated in the presence of numerical diffusion (very significantly in some cases) and will be overestimated (but not significantly) in the presence of numerical dispersion, assuming numerical diffusion is absent.

\section{Conclusions}

Five different numerical methods were applied to synthetic solute concentration data under the same grid resolutions and non-dimensional numerical conditions, and optimised dispersion coefficients and velocities were obtained. The ranges of advection number, dispersion number and Peclet number covered were 0.900-0.113, 0.600-0.009 and 1.5-12.0, respectively. For $P_{e}<5$, using the optimised dispersion coefficients and velocities, all the numerical methods were able to simulate the synthetic data well (sum of squared errors being $<0.01 \mu^{2} / 1^{2}$ and with little visual difference between synthetic and simulated concentration profiles), but simulations were increasingly inaccurate as $P_{e}$ increased beyond 5 . However, optimum model fits to the synthetic data were only achieved by adjusting, to greater or lesser extents, the velocity and dispersion coefficient to accommodate the presence of numerical errors. Differences between the optimised and synthetic velocity values were typically less than $2 \%$ for $P_{e}<5$, but increased to about $8 \%$ for $P_{e}=12$. The methods yielded a range of optimal values of the dispersion coefficient some of which were significantly different to the value used to generate the synthetic data. The behaviour of the methods was generally consistent with the known 
presence of artificial mixing, caused by numerical diffusion and/or numerical dispersion, in them. In this regard, numerical diffusion in Backward-Time/Centred-Space and Implicit QUICK reduced the optimised dispersion coefficient from $0.75 \mathrm{~m}^{2} / \mathrm{s}$ to about $0.25 \mathrm{~m}^{2} / \mathrm{s}$ for $P_{e}<5$, whilst optimisations with Crank-Nicolson, MacCormack and QUICKEST, which contain no numerical diffusion, produced reliable optimised dispersion coefficients in this $P_{e}$ range. For $P_{e}>5$ the simulations from all five methods were affected by an oscillatory distortion which became more prominent with increasing $P_{e}$, this being generally consistent with the known presence of numerical dispersion in the methods. In this higher $P_{e}$ range optimised dispersion coefficients for Crank-Nicolson, MacCormack and Backward-Time/Centred-Space increased a little from the values in the lower $P_{e}$ range due to the fitting of the distorted simulated concentration profile to the whole synthetic one, whilst those from Implicit QUICK and QUICKEST showed a substantial decrease from the value in the lower $P_{e}$ range due to numerical diffusion. It was suggested that results for these two methods were affected by numerical errors from higher-order truncation error terms than the second and third spatial derivatives.
Acknowledgements The authors would like to thank an anonymous reviewer for suggesting that an improved interpretation of the results could be achieved by using the modified equation approach to highlight the nature of the numerical errors in the five methods used in the work.

\section{Compliance with ethical standards}

Conflict of interest The authors declare that they have no conflict of interest.

Open Access This article is distributed under the terms of the Creative Commons Attribution 4.0 International License (http://creativeco mmons.org/licenses/by/4.0/), which permits unrestricted use, distribution, and reproduction in any medium, provided you give appropriate credit to the original author(s) and the source, provide a link to the Creative Commons license, and indicate if changes were made.

\section{Appendix 1}

See Figs. 8 and 9 .
Fig. 8 Computational grid for a finite difference method: information at the nodes is used directly to express solute concentration derivatives
Time, $\mathrm{t}$

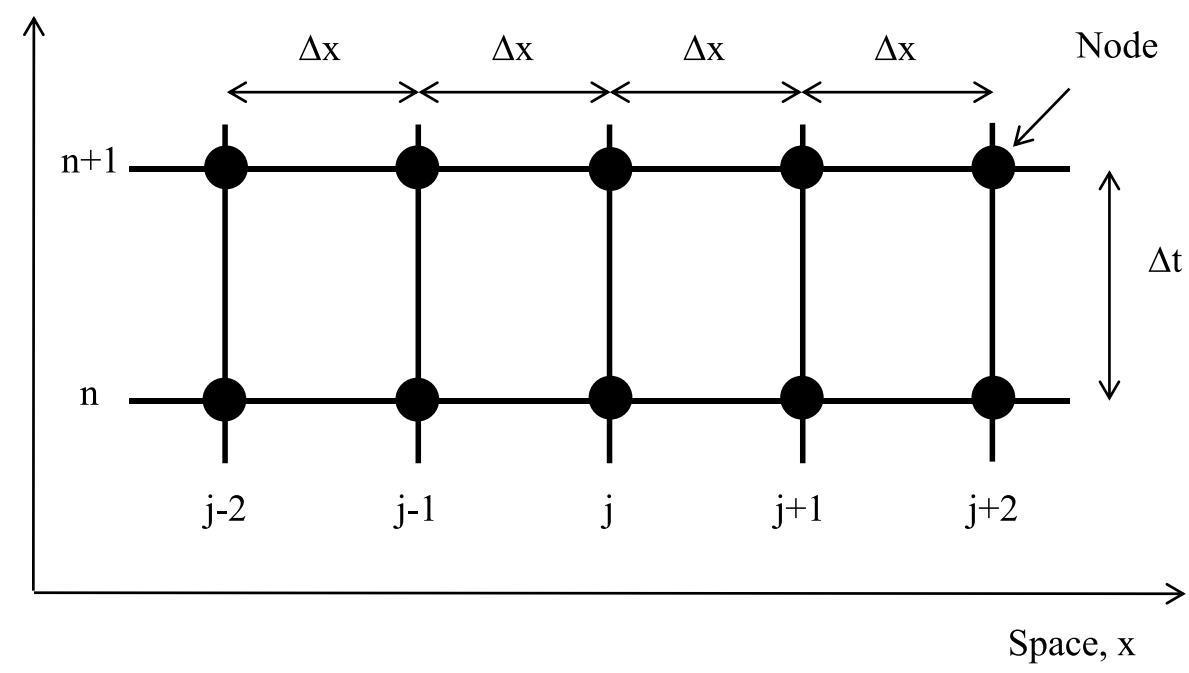


Fig. 9 Computational grid for a finite volume method: advective and dispersive solute fluxes passing through the control volume faces are interpolated from information at the nodes
Time, $\mathrm{t}$

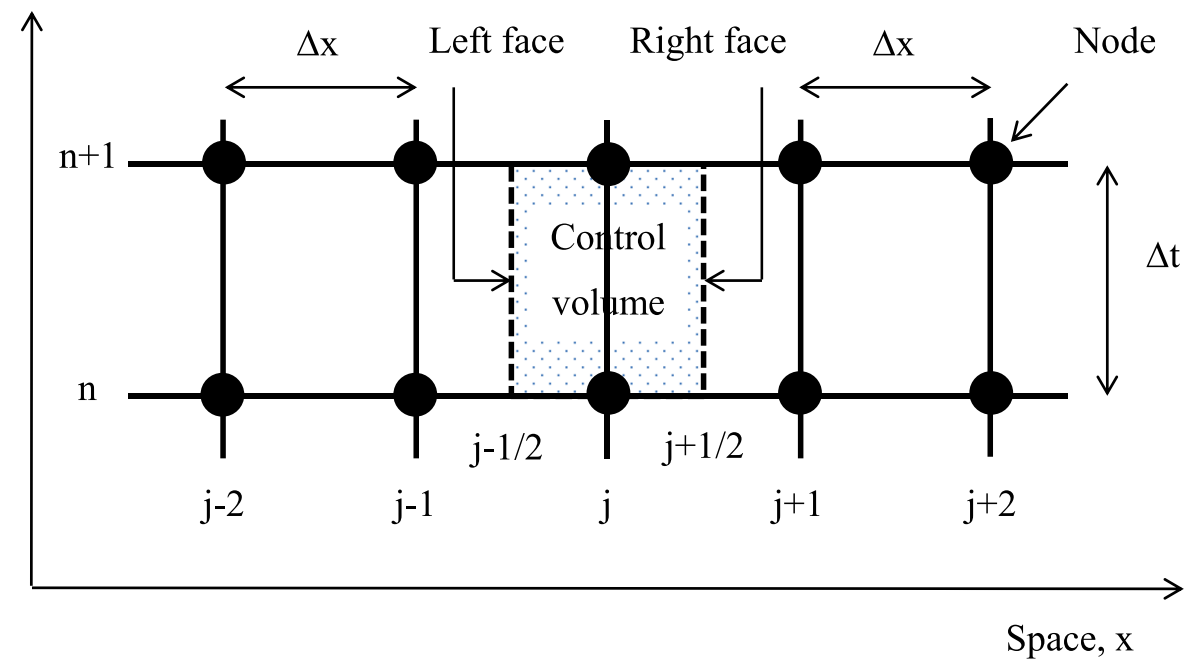

\section{Appendix 2}

Following Szymkiewicz (2010), the modified equation approach aims to write a numerical method in the following form:

$\varphi_{t}+v \varphi_{x}=D_{n} \varphi_{x x}+E_{n} \varphi_{x x x}+\cdots$

where $\varphi_{t}$, represents the first temporal concentration derivative and $\varphi_{x}, \varphi_{x x}$ and $\varphi_{x x x}$ represent the first, second and third spatial concentration derivatives. $D_{n}$ and $E_{n}$ are termed the coefficients of numerical diffusion and numerical dispersion, respectively. Only advection is considered because it is only truncation errors emanating from the first temporal and first spatial derivatives that contribute to the second and third spatial derivative terms in Eq. (28). By using Taylor series expansions to express a nodal concentration in terms of a neighbouring nodal concentration that is typically separated from it by one space step $(\Delta x)$ or one time step $(\Delta t)$, the following expressions can be obtained for the five numerical methods used in the paper.

Backward-Time/Centred-Space

$\varphi_{t}-\frac{\Delta t}{2} \varphi_{t t}+\frac{\Delta t^{2}}{6} \varphi_{t t t}+v\left(\varphi_{x}+\frac{\Delta x^{2}}{6} \varphi_{x x x}\right)+\cdots=0$

\section{Crank-Nicolson}

$$
\begin{aligned}
\varphi_{t} & +\frac{\Delta t}{2} \varphi_{t t}+\frac{\Delta t^{2}}{6} \varphi_{t t t} \\
& +v\left(\varphi_{x}+\frac{\Delta x^{2}}{6} \varphi_{x x x}+\Delta t \varphi_{t x}+\frac{\Delta t^{2}}{2} \varphi_{t t x}\right)+\cdots=0
\end{aligned}
$$

\section{Implicit QUICK}

$$
\varphi_{t}-\frac{\Delta t}{2} \varphi_{t t}+\frac{\Delta t^{2}}{6} \varphi_{t t t}+v\left(\varphi_{x}+\frac{\Delta x^{2}}{24} \varphi_{x x x}\right)+\cdots=0
$$

MacCormack

$$
\begin{aligned}
\varphi_{t} & +\frac{\Delta t}{2} \varphi_{t t}+\frac{\Delta t^{2}}{6} \varphi_{t t t}+v\left(\varphi_{x}+\frac{\Delta x^{2}}{6} \varphi_{x x x}\right) \\
& +\frac{c}{2}\left(\Delta x \varphi_{x t}-\frac{\Delta x^{2}}{2} \varphi_{x x t}+\frac{\Delta t \Delta x}{2} \varphi_{x t t}\right)+\cdots=0
\end{aligned}
$$

\section{QUICKEST}

$\varphi_{t}+\frac{\Delta t}{2} \varphi_{t t}+\frac{\Delta t^{2}}{6} \varphi_{t t t}+v\left(\varphi_{x}-\frac{\Delta x c}{2} \varphi_{x x}+\frac{\Delta x^{2}}{6} c^{2} \varphi_{x x x}\right)+\cdots=0$

where $\mathrm{c}$ is the advection number, as previously defined (Eq. 8). Note that the time-centred methods contain crossderivatives $\left(\varphi_{x t}, \varphi_{t t x}, \varphi_{x x t}\right)$ as a result of the need to express everything in terms of a concentration and its derivatives at the same node. This involves using Taylor series expansions of concentration derivatives in exactly the same way as Taylor series expansions are used to express concentrations. Finally use is made of the following relationships, obtained by differentiating the advection equation (Szymkiewicz 2010), to replace all temporal derivatives and cross-derivatives:

$\varphi_{t t}=v^{2} \varphi_{x x}$

$\varphi_{t t t}=-v^{3} \varphi_{x x x}$

$\varphi_{t x}=\varphi_{x t}=-v \varphi_{x x}$

$\varphi_{x x t}=-v \varphi_{x x x}$

$\varphi_{t t x}=\varphi_{x t t}=v^{2} \varphi_{x x x}$

The analysis is completed below for two of the methods. Implicit QUICK 
Equation (31) becomes:

$\varphi_{t}-\frac{\Delta t v^{2}}{2} \varphi_{x x}-\frac{\Delta t^{2} v^{3}}{6} \varphi_{x x x}+v\left(\varphi_{x}+\frac{\Delta x^{2}}{24} \varphi_{x x x}\right)+\cdots=0$

Thus:

$\varphi_{t}+v \varphi_{x}=\frac{\Delta t v^{2}}{2} \varphi_{x x}-\frac{\Delta \mathrm{x}^{2} v}{6}\left(-c^{2}+\frac{1}{4}\right) \varphi_{x x x}+\cdots$

MacCormack

Equation (32) becomes:

$$
\begin{aligned}
\varphi_{t} & +\frac{\Delta t v^{2}}{2} \varphi_{x x}-\frac{\Delta t^{2} v^{3}}{6} \varphi_{x x x}+v\left(\varphi_{x}+\frac{\Delta x^{2}}{6} \varphi_{x x x}\right) \\
& +\frac{c}{2}\left(-\Delta x v \varphi_{x x}+\frac{\Delta x^{2} v}{2} \varphi_{x x x}+\frac{\Delta t \Delta x v^{2}}{2} \varphi_{x x x}\right)+\cdots=0
\end{aligned}
$$

Thus:

$$
\begin{aligned}
\varphi_{t}+v \varphi_{x}= & -\frac{\Delta t v^{2}}{2} \varphi_{x x}+\frac{\Delta t^{2} v^{3}}{6} \varphi_{x x x}-\frac{\Delta x^{2} v}{6} \varphi_{x x x} \\
& +\frac{c}{2} \Delta x v \varphi_{x x}-\frac{\Delta x^{2} v c}{4} \varphi_{x x x}-\frac{\Delta t \Delta x v^{2} c}{4} \varphi_{x x x}+\cdots \\
\varphi_{t}+v \varphi_{x}= & -\frac{\Delta t v^{2}}{2} \varphi_{x x}+\frac{\Delta t^{2} v^{3}}{6} \varphi_{x x x}-\frac{\Delta x^{2} v}{6} \varphi_{x x x} \\
& +\frac{\Delta t v^{2}}{2} \varphi_{x x}-\frac{\Delta t \Delta x v^{2}}{4} \varphi_{x x x}-\frac{\Delta t^{2} v^{3}}{4} \varphi_{x x x}+\cdots \\
\varphi_{t}+v \varphi_{x}= & -\frac{\Delta x^{2} v}{12}\left(c^{2}+3 c+2\right) \varphi_{x x x}
\end{aligned}
$$

\section{Appendix 3}

The algorithm for any implicit method involving three unknown nodal concentrations surrounding the $j$ th node can be written in the following form:

$\alpha \varphi_{j-1}+\beta \varphi_{j}+\gamma \varphi_{j+1}=\delta_{j}$

where $\alpha, \beta$ and $\gamma$ are constant functions of time step, space step, velocity and dispersion coefficient, $\delta_{j}$ is a spatially varying function of time step, space step, velocity, dispersion coefficient and known concentration values (at time level, $n$ ) and $\varphi_{j-1}, \varphi_{j}$ and $\varphi_{j+1}$ are unknown concentrations (at time level $n+1)$. Letting $j=2$ represent the first internal node and recognising that $\varphi_{1}$ is known from the upstream boundary condition, Eq. (39) can be written as:

$p_{2} \varphi_{2}+q_{3} \varphi_{3}=r_{2}$

where $p_{2}, q_{3}$ and $r_{2}$ are functions of $\alpha, \beta, \gamma, \delta_{2}$ and $\varphi_{1}$. For $j=3$, Eq. (39) can be written as:

$p_{3} \varphi_{3}+q_{4} \varphi_{4}=r_{3}$ where $\varphi_{2}$ has been eliminated using Eq. (40). Repeating this process for successively increasing $j$, we eventually obtain:

$p_{m-1} \varphi_{m-1}+q_{m} \varphi_{m}=r_{m-1}$

where $\varphi_{m}$ is the known concentration at the downstream boundary (at time level $n+1$ ) and $p_{m-1}, q_{m}$ and $r_{m-1}$ are given by:

$p_{m-1}=\frac{-\alpha q_{m-1}}{p_{m-2}}+\beta$

$q_{m}=\gamma$

$r_{m-1}=\delta_{m-1}-\frac{\alpha r_{m-2}}{p_{m-2}}$

The solution is summarised by the following three steps, which are repeated for every time step:

Calculate $\alpha, \beta, \gamma$ and, for $j=2$ to $m-1, \delta_{j}$ : then calculate $p_{2}(=\beta), q_{3}(=\gamma)$ and $r_{2}\left(=\delta_{2}-\alpha \varphi_{1}\right)$.

Undertake a forward sweep: for $j=4$ to $m-1$ calculate $p_{j-1}, q_{j}$ and $r_{j-1}$ [using Eqs. (43)-(45)].

Undertake a backward sweep: for $j=m-1$ to 2 calculate $\varphi_{j}$ [using Eq. (42)].

It is straightforward to derive a similar solution for an implicit method containing a fourth unknown nodal concentration (e.g. $\varphi_{j-2}$ needs to be included in Eq. (39) for Implicit QUICK) assuming that both the boundary value and the value at the first interior node are known. In this, Eqs. (42) and (44) remain the same, but Eqs. (43) and (45) are modified.

\section{References}

Abbott MB, Basco DR (1989) Computational fluid dynamics: an introduction for engineers. Longman Scientific \& Technical, Harlow

Ani E-C, Wallis S, Kraslawski A, Agachi S (2009) Development, calibration and evaluation of two mathematical models for pollutant transport in a small river. Environ Model Softw 24:1139_ 1152. https://doi.org/10.1016/j.envsoft.2009.03.008

Barnett AG (1983) Exact and approximate solutions of the advection-dispersion equation. In: Proceedings of the 20th IAHR Congress, Moscow, vol 3, pp 180-190

Bennett ND et al (2013) Characterising performance of environmental models. Environ Model Softw 40:1-20. https://doi. org/10.1016/j.envsoft.2012.09.011

Billo EJ (2007) Excel for scientists and engineers: numerical methods. Wiley, Hoboken

Chanson H (2004) Enviromental hydraulics for open channel flows. Elsevier, Oxford

Chapra SC (2008) Surface water-quality modeling. Waveland Press, Long Grove

Chapra SC, Canale RP (2015) Numerical methods for engineers. McGraw-Hill Education, New York 
Chin DA (2013) Water-quality engineering in natural systems: fate and transport processes in the water environment. Wiley, Hoboken

Ferziger JH, Peric M (2002) Computational methods for fluid dynamics. Springer, Berlin

Fischer HB, List EJ, Koh RCY, Imberger J, Brooks NH (1979) Mixing in inland and coastal waters. Academic Press, San Diego

Fürst J, Furmánek P (2011) An implicit MacCormack scheme for unsteady flow calculations. Comput Fluids 46:231-236. https ://doi.org/10.1016/j.compfluid.2010.09.036

Fylstra D, Lasdon L, Watson J, Allan W (1998) Design and use of the Microsoft Excel solver. Interfaces 28:9-55

Graf WH, Altinakar MS (1998) Fluvial hydraulics: flow and transport processes in channels of simple geometry. Wiley, Chichester

Hayase T, Humphrey JAC, Greif R (1992) A consistently formulated QUICK scheme for fast and stable convergence using finite-volume iterative calculation procedures. J Comput Phys 98:108-118

Hoffman JD (2001) Numerical methods for engineers and scientists. Marcel Dekker, New York

Karahan H (2006) Implicit finite difference techniques for the advection-diffusion equation using spreadsheets. Adv Eng Softw 37:601-608

Karahan H (2007) Unconditional stable explicit finite difference technique for the advection-diffusion equation using spreadsheets. Adv Eng Softw 38:80-86. https://doi.org/10.1016/j. advengsoft.2006.08.001

Karahan H (2008) Solution of weighted finite difference techniques with the advection-diffusion equation using spreadsheets. Comput Appl Eng Educ 16:147-156. https://doi.org/10.1002/cae.20140

Kumar A, Jaiswal DK, Kumar N (2009) Analytical solutions of onedimensional advection-diffusion equation with variable coefficients in a finite domain. J Earth Syst Sci 118:539-549

Lasdon LS, Waren AD, Jain A, Ratner N (1978) Design and testing of a generalized reduced gradient code for nonlinear programming. ACM Trans Math Softw 4:34-50

Leonard BP (1979) A stable and accurate convective modelling procedure based on quadratic upstream interpolation. Comput Methods Appl Mech Eng 19:59-98. https://doi. org/10.1016/0045-7825(79)90034-3

MacCormack RW (1982) A numerical method for solving the equations of compressible viscous flow. AIAA J 20:1275-1281

Manson JR, Wallis SG (1995) An accurate numerical algorithm for advective transport. Commun Numer Methods Eng 11:1039-1045

Manson JR, Wallis SG, Hope D (2001) A conservative semi-Lagrangian transport model for rivers with transient storage zones. Water Resour Res 37:3321-3329. https://doi.org/10.1029/2001WR0002 30

Martin JL, McCutcheon SC (1999) Hydrodynamics and transport for water quality modeling. CRC Press, Boca Rotan
Ogata A, Banks RB (1961) A solution of the differential equation of longitudinal dispersion in porous media. Geological Survey Professional Paper 411-A, US Department of Interior, Washington, USA

Runkel RL, Broshears RE (1991) One-dimensional transport with inflow and storage (OTIS): a solute transport model for small streams. CADSWES, Department of Civil, Environmental and Architectural Engineering, University of Colorado, Boulder

Rutherford JC (1994) River mixing. Wiley, Chichester

Semuwemba J (2011) Modelling tracer breakthrough curves to determine stream reaeration and hydrodynamic properties. Ph.D. thesis, Queens University Belfast, UK

Singh SK, Beck MB (2003) Dispersion coefficient of streams from tracer experiment data. J Environ Eng ASCE 129:539-546. https ://doi.org/10.1061/(ASCE)0733-9372(2003)129:6(539)

Sobey RJ (1984) Numerical alternatives in transient stream response. J Hydraul Eng ASCE 110:749-772

Szymkiewicz R (2010) Numerical modelling in open channel hydraulics. Water science and technology library, vol 83. Springer, Berlin

Taylor GI (1954) The dispersion of matter in turbulent flow through a pipe. Proc R Soc Lond A223:446-468

USEPA (2009) Guidance on the development, evaluation and application of environmental models. NSCEP, Cincinnati

Vaghela CR, Vaghela AR (2014) Synthetic flow generation. Int J Eng Res Appl 4:66-71

van den Bos A (2007) Parameter estimation for scientists and engineers. Wiley, Hoboken. https://doi.org/10.1002/9780470173862

Versteeg HK, Malalasekera W (2007) An introduction to computational fluid dynamics: the finite volume method. Pearson Education Ltd, Harlow

Wallis SG (2007) The numerical solution of the advection-dispersion equation: a review of some basic principles. Acta Geophys 55:85-94. https://doi.org/10.2478/s11600-006-0044-5

Wallis SG, Manson JR (1997) Accurate numerical simulation of advection using large time steps. Int J Numer Methods Fluids 24:127-139

Wallis SG, Manson JR (2004) Methods for predicting dispersion coefficients in rivers. Water Manag ICE 157:131-141. https://doi. org/10.1680/wama.2004.157.3.131

Wallis SG, Manson JR, Filippi L (1998) A conservative semi-Lagrangian algorithm for one-dimensional advection-diffusion. Commun Numer Methods Eng 14:671-679

Wallis SG, Osuch M, Manson JR, Romanowicz R, Demars BOL (2013) On the estimation of solute transport parameters for rivers. In: Rowiński P (ed) Experimental and computational solutions of hydraulic problems. Springer, Berlin, pp 415-425. https://doi. org/10.1007/978-3-642-30209-1_30 